\title{
Assessment and Correction of on-Orbit Radiometric Calibration for FY-3 VIRR Thermal Infrared Channels
}

\author{
Na Xu ${ }^{1,2, *}$, Lin Chen ${ }^{1,2}$, Xiuqing Hu ${ }^{1,2}$, Liyang Zhang ${ }^{2}$ and Peng Zhang ${ }^{2}$
}

1 Key Laboratory of Radiometric Calibration and Validation for Environmental Satellites, China Meteorological Administration, Beijing 100081, China; E-Mails: chenlin@cma.gov.cn (L.C.); huxq@cma.gov.cn (X.H.)

2 National Satellite Meteorological Center, China Meteorological Administration, Beijing 100081, China; E-Mails: zhangly@cma.gov.cn (L.Z.); zhangp@cma.gov.cn (P.Z.)

* Author to whom correspondence should be addressed; E-Mail: xuna@cma.gov.cn; Tel.: +86-10-6840-6704.

Received: 13 September 2013; in revised form: 25 February 2014 / Accepted: 27 February 2014 / Published: 28 March 2014

\begin{abstract}
FengYun-3 (FY-3) Visible Infrared Radiometer (VIRR), along with its predecessor, Multispectral Visible Infrared Scanning Radiometer (MVISR), onboard FY-1C\&D have had continuous global observation more than 14 years. This data record is valuable for weather prediction, climate monitoring, and environment research. Data quality is vital for satellite data assimilations in Numerical Weather Prediction (NWP) and quantitative remote sensing applications. In this paper, the accuracies of radiometric calibration for VIRR onboard FY-3A and FY-3B, in thermal infrared (TIR) channels, are evaluated using the Low Earth Orbit (LEO)-LEO simultaneous nadir overpass intercalibration method. Hyperspectral and high-quality observations from Infrared Atmosphere Sounding Instrument (IASI) onboard METOP-A are used as reference. The biases of VIRR measurements with respect to IASI over one-and-a-half years indicate that the TIR calibration accuracy of FY-3B VIRR is better than that of FY-3A VIRR. The brightness temperature (BT) measured by FY-3A/VIRR is cooler than that measured by IASI with monthly mean biases ranging from $-2 \mathrm{~K}$ to $-1 \mathrm{~K}$ for channel 4 and $-1 \mathrm{~K}$ to $0.2 \mathrm{~K}$ for channel 5. Measurements from FY-3B/VIRR are more consistent with that from IASI, and the annual mean biases are $0.84 \pm 0.16 \mathrm{~K}$ and $-0.66 \pm 0.18 \mathrm{~K}$ for channels 4 and 5 , respectively. The BT biases of FY-3A/VIRR show scene temperature-dependence and seasonal variation, which are not found from FY-3B/VIRR BT biases. The temperature-dependent biases are shown to be attributed to the nonlinearity of detectors.
\end{abstract}


New nonlinear correction coefficients of FY-3A/VIRR TIR channels are reevaluated using various collocation samples. Verification results indicate that the use of the new nonlinear correction can greatly correct the scene temperature-dependent and systematic biases.

Keywords: FengYun-3; visible and infrared radiometer; hyperspectral; Infrared Atmosphere Sounding Instrument (IASI); intercalibration; nonlinear correction

\section{Introduction}

FengYun-3 (FY-3) series is the second-generation Chinese polar-orbiting meteorological satellite. The first batch consists of two experimental spacecraft (FY-3A\&B) that were launched on 27 May 2008, and 5 November 2011, respectively. FY-3A is a morning orbit satellite with an equatorial crossing time of 10:30 a.m., whereas FY-3B is an afternoon orbit satellite with an equatorial crossing time of 1:30 p.m. The Visible and InfraRed Radiometer (VIRR) is an important payload onboard FY-3A\&B. It has 10 channels covering the spectral range, from visible (VIS) to thermal infrared (TIR), and the channel specification is illustrated in Table D1 of [1]. The VIRR makes operational observations on FY-3A\&B, as it is a heritage instrument from Multispectral Visible Infrared Scanning Radiometer (MVISR) onboard FY-1C\&D [1]. VIRR is the only instrument on the current FY-3 platform that can provide high spatial resolution and narrow-band infrared window measurements, which are vital for cloud or fog detection and quantitative retrieval of cloud properties, surface temperature, and Earth radiation budget. With over 14 years of continuous global observation, data records from VIRR and MVISR can be used for environment monitoring and numerical weather prediction (NWP) assimilation, and, also, serve as a valuable data set for climate change detection. All these applications have strong requirements for accurate satellite radiance measurements. Therefore, independently assessing and monitoring the performance of on-orbit radiometric calibration, which can help with understanding the data quality and further improving calibration, is necessary.

Intercalibration against a well-calibrated instrument at Low Earth Orbit (LEO) is a common method that has been widely used to assess the data quality as it can provide frequent evaluation results [2]. Under the framework of the Global Space-based Intercalibration System (GSICS) project [3-5], hyperspectral instruments Infrared Atmosphere Sounding Instrument (IASI) and Atmospheric Infrared Sounder (AIRS) have shown to be the best reference for onboard calibration evaluation in the TIR region. IASI is a Fourier transform spectrometer onboard the METOP satellite, with 8461 channels covering a spectral range from $3.7 \mu \mathrm{m}$ to $15.5 \mu \mathrm{m}$. AIRS is a grating spectrometer onboard the AQUA satellite, with 2378 channels covering the spectral range from $3.7 \mu \mathrm{m}$ to $15.4 \mu \mathrm{m}$, but with some gaps. Both IASI and AIRS achieve good radiometric and spectral calibration performance [6,7], and the spectral radiance measurements from these two instruments are quite consistent with a difference of less than $0.1 \mathrm{~K}$ [8]. Based on these two references, the IR calibration behaviors of global geostationary (GEO) imagers operated by different agencies have been widely evaluated [9-14].

In this study, the on-orbit calibration accuracies of FY-3A\&B VIRR TIR channels are independently assessed using the LEO-LEO simultaneous nadir overpass (SNO) intercalibration method. Based on SNO collocated data between VIRR and IASI over one-and-a-half years, the brightness temperature 
(BT) biases of VIRR, including their scene temperature-dependence and seasonal variation characters were investigated. According to collocated samples, new nonlinear correction coefficients were reevaluated to reduce calibration bias. Considering its high resolution and continuous spectral character, IASI, onboard METOP-A, is chosen as the reference instrument in this paper. Figure 1 displays the simulated top-of-atmosphere (TOA) typical spectra of IASI, as well as the normalized spectral response functions (SRFs) of FY-3A\&B VIRR TIR channels. VIRR has two TIR channels and are all located at the atmospheric window region (i.e., $\mathrm{CH} 4$ and $\mathrm{CH} 5$ ), which can be fully covered by the IASI spectra.

Figure 1. Normalized spectral response functions of FY-3A\&B VIRR thermal infrared channels (CH4 and $\mathrm{CH} 5)$, as well as the simulated top-of-atmosphere typical spectra of IASI over a clear sky. Solid lines denote SRFs of channel 4, and dashed lines denote that of channel 5. Black and red lines are for FY-3A and FY-3B, respectively.

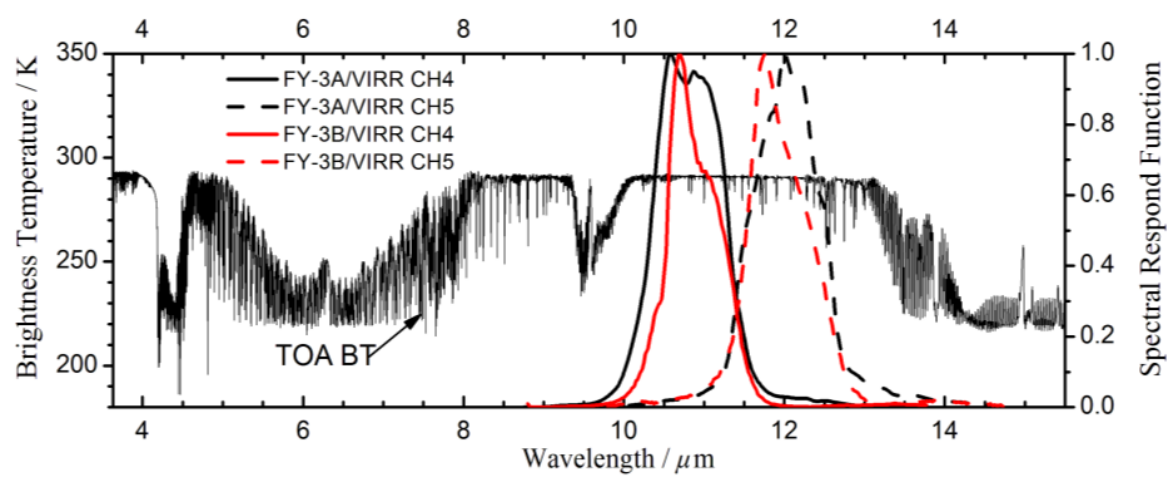

This paper is organized as follows: Section 2 describes the intercalibration method and satellite data used here. Section 3 is evaluation and analysis results of the BT biases of VIRR against IASI. Section 4 shows recalculation and verification results of nonlinear correction coefficients for FY-3A/VIRR, based on collocation samples over five months. Finally, conclusions are given in Section 5.

\section{Methodology}

\subsection{LEO-LEO Intercalibration}

Different instruments on LEO spacecraft with similar spectral regions can be compared using their simultaneous measurements over the same location. This approach is called the simultaneous nadir overpass (SNO) intercalibration method. The on-orbit calibration accuracies of these instruments can be assessed using a well-calibrated instrument as reference. The key factors of this method are to ensure the consistency of spectral responses and the observation conditions between the two instruments. In this paper, VIRR measurements are compared with the simulated radiances derived by convolving hyperspectral observations of IASI with VIRR SRFs. The IASI spectra can fully cover the two TIR channels of VIRR as shown in Figure 1, thus, the difference in spectra can be neglected.

A similar method developed for GEO-LEO intercalibration $[4,5,9,12]$ is applied in this study, and some differences in collocation criteria occur when considering LEO-LEO special characteristics. Four main steps are used in LEO-LEO SNO intercalibration. 
(1) Data collection: According to the prediction of satellite nadir tracks, orbit cross points of the two satellites are selected when their nadir overpass the same target within $10 \mathrm{~min}$. According to the overpass time, the corresponding radiance products of VIRR and IASI are downloaded.

(2) Observation collocation: The spatial and temporal collocated pixel pairs with similar view geometries are essential to intercalibration. Three collocation criteria are used to obtain the SNO samples. The nadir resolution of VIRR is approximately $1.1 \mathrm{~km}$, such that the spatial collocation threshold defined as the distance between centers of pixel pairs is set to less than $1.5 \mathrm{~km}$. The time difference of observations is limited to less than $5 \mathrm{~min}$. View geometry collocation ensures a similar atmospheric optical path that is dependent on the satellite zenith and azimuth angles. In this work, the cosine of the satellite viewing zenith angle is used for geometry collocation, and the criterion threshold is defined as $\mid \cos \left(\mathrm{FY} \_z e n\right) / \cos (\mathrm{REF}$ zen $)-1 \mid<5 \%$. The bi-direction characterization of ground surface in the TIR spectra has less effect on the emitted radiance, so the satellite azimuth angle difference is only roughly restricted to less than $90^{\circ}$. The nearby collocated pixels are simultaneously observing the same target, such that the solar angle differences can be ignored.

(3) Data transformation: Hyperspectral measurements are convolved with SRFs to derive IASI-simulated radiances corresponding to VIRR channels. Regarding to the large difference in spatial resolution, VIRR measurements with high spatial resolutions are averaged and degraded to match the large instantaneous field of view (IFOV) pixels of IASI with lower resolutions (approximately $12 \mathrm{~km}$ at nadir). Radiances from these matched footprints are supposed to be consistent, such that spatially homogeneous footprints are selected. In this work, the relative standard deviation of $13 \times 13$ VIRR pixels over IASI IFOV is used as a homogeneous scene criterion, i.e., $\left(\operatorname{StdRad}_{\mathrm{FY}} / \mathrm{MeanRad} \mathrm{FY}_{\mathrm{FY}}\right)_{\mathrm{IASI} \mathrm{IFOV}}<0.5 \%$.

(4) Radiance comparison: In the TIR spectra, calibration assessments are generally based on BT. Collocated radiances are converted to BT using pre-created lookup tables corresponding to VIRR channels, and calibration bias of VIRR is assessed using IASI-simulated BT as reference.

\subsection{Data}

Sufficient collocation samples are required to ensure reliable evaluation results. Figure 2 displays the dates when SNO cross points between FY-3A\&B and METOP-A (overpass time difference within $10 \mathrm{~min}$ ) arise, based on their nadir tracks in 2012. The SNO cross points generally appear every other month, periodically, and last for approximately 10 days during each period. Figures 3 and 4 show an example of spatial distributions of all SNO cross points, respectively, for FY-3A vs. METOP-A and FY-3B vs. METOP-A pairs. The SNO cross points mostly occur in relatively high latitudes of $83^{\circ}$ or $74^{\circ}$ in both the northern and southern hemispheres, respectively, for the two satellite pairs. These cross points enable the collection of sufficient collocation samples, thus, ensuring the reliability of assessment results. 
Figure 2. Temporal distributions of cross points between FY-3A\&B and METOP-A nadir tracks during 2012. The difference in overpass time is within $10 \mathrm{~min}$.

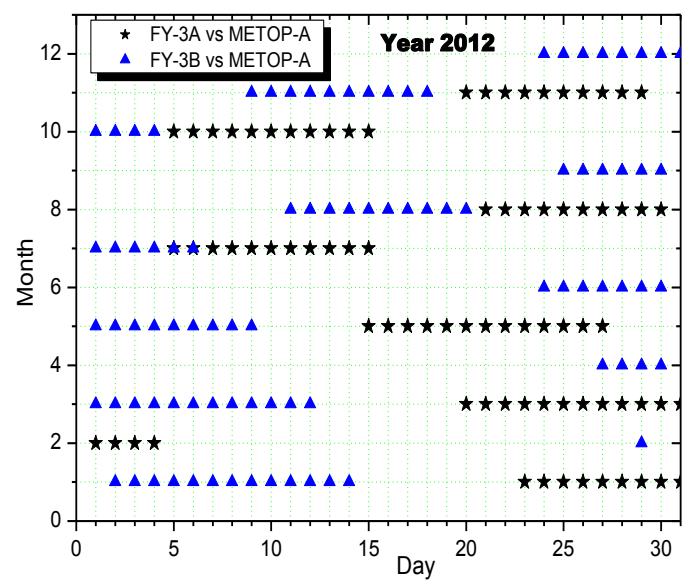

Figure 3. Spatial distributions of cross points between FY-3A and METOP-A nadir tracks in one day. The difference in overpass time is within $10 \mathrm{~min}$.
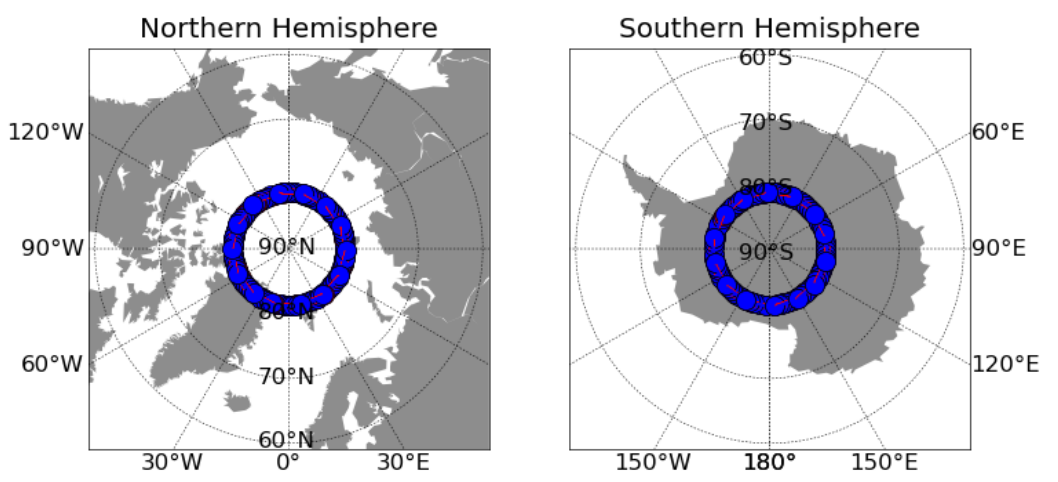

Red line: FENGYUN-3A Blue line: METOP-A

Figure 4. Spatial distributions of cross points between FY-3B and METOP-A nadir tracks in one day. The difference in overpass time is within $10 \mathrm{~min}$.
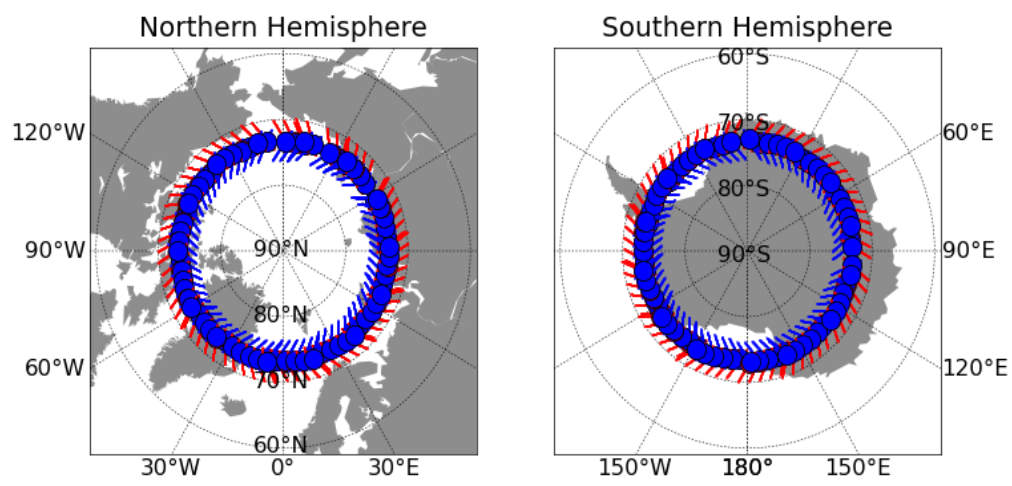

Red line: FENGYUN-3B Blue line: METOP-A

\subsection{Uncertainty Analysis}

To obtain sufficient samples under various sky conditions, a set of collocation approximates is applied using several criteria described in Section 2.1, which may cause systematic errors in the 
calibration assessment. Using thousands of collocation samples from August to December 2012, the collocation uncertainty is investigated based on BT differences (BT Difs) between VIRR and IASI. Taking FY-3B/VIRR as an example, Figures 5 and 6 are the scatterplots of BT Difs in CH4 and CH5 varying with four main collocation factors: observation time difference, view geometric difference (zenith and azimuth angles), and scene spatial homogeneity. These BT Difs are separately fitted as a function of corresponding collocation factors, and the fitting results are drawn in solid lines. The linear fitting lines are nearly parallel to the x-axis, which indicates that BT Difs are not dependent on any of these collocation factors.

Figure 5. Scatterplots of BT differences between FY-3B/VIRR channel 4 and IASI varying with observation time difference (a); satellite zenith angle (b); target homogeneity (c); and satellite azimuth angle (d) based on collocated samples from August to December in 2012 (Solid lines are linear fitting results).
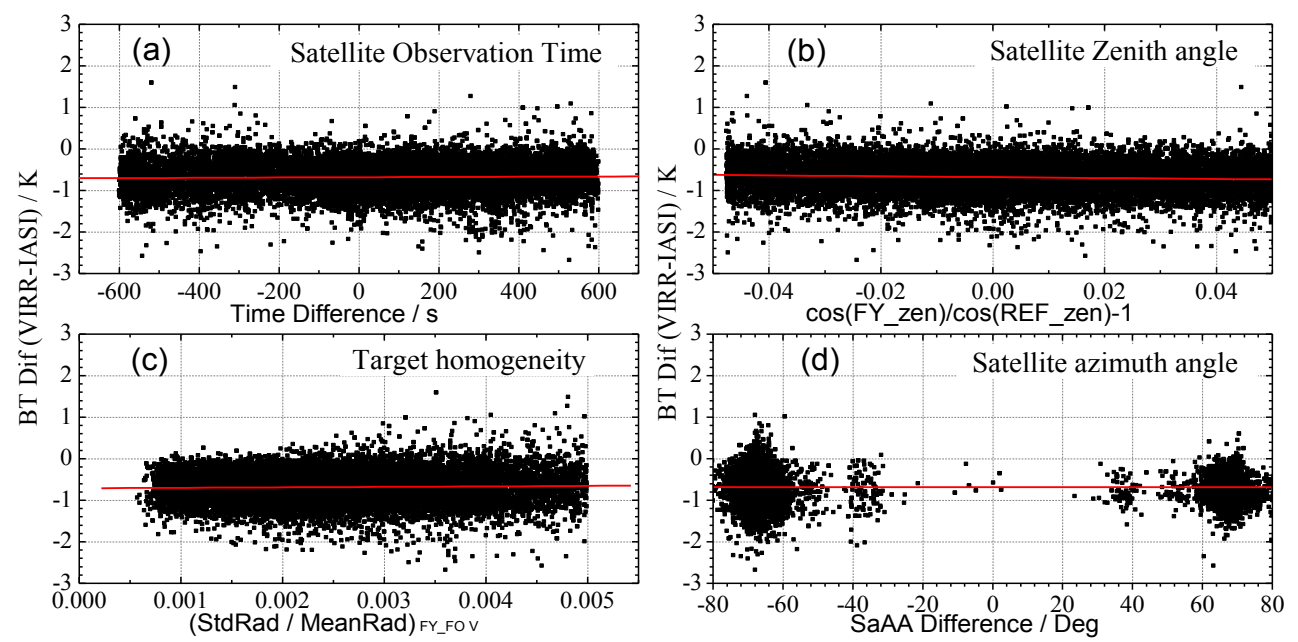

Figure 6. Scatterplots of BT differences between FY-3B/VIRR channel 5 and IASI varying with observation time difference (a); satellite zenith angle (b); target homogeneity (c); and satellite azimuth angle (d) based on collocated samples from August to December in 2012 (Solid lines are linear fitting results).
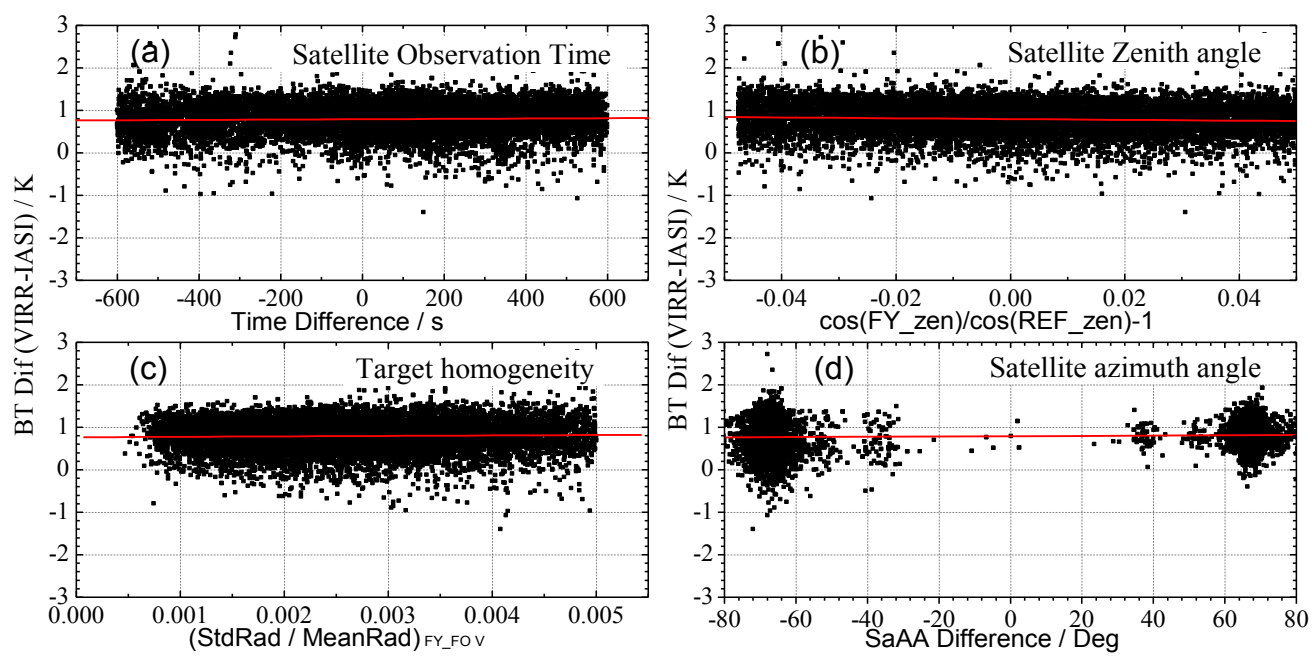


\section{Assessment Results}

\subsection{Scene Temperature Dependence}

Figures 7 and 8 show radiance comparisons between VIRR and IASI in August 2012 (A), as well as their observation differences in radiances (B) and bright temperatures (C). Taking IASI measurements as a reference, the observation difference indicates VIRR calibration bias. Thousands of collocation pairs in these figures show good correlation relationships, with correlation coefficients better than 0.9997. This finding reveals that calibration biases of VIRR TIR channels during this month are quite stable and that the collocation samples used in this work are reliable.

Figure 7. Radiance comparison between FY-3A/VIRR and METOP-A/IASI (a); as well as their observation differences (VIRR-IASI) in radiance (b) and BT (c) using collocation samples in August 2012. The red lines are quadratic fitting results. The top plots are for $\mathrm{CH} 4$, and those on the bottom are for $\mathrm{CH} 5$.
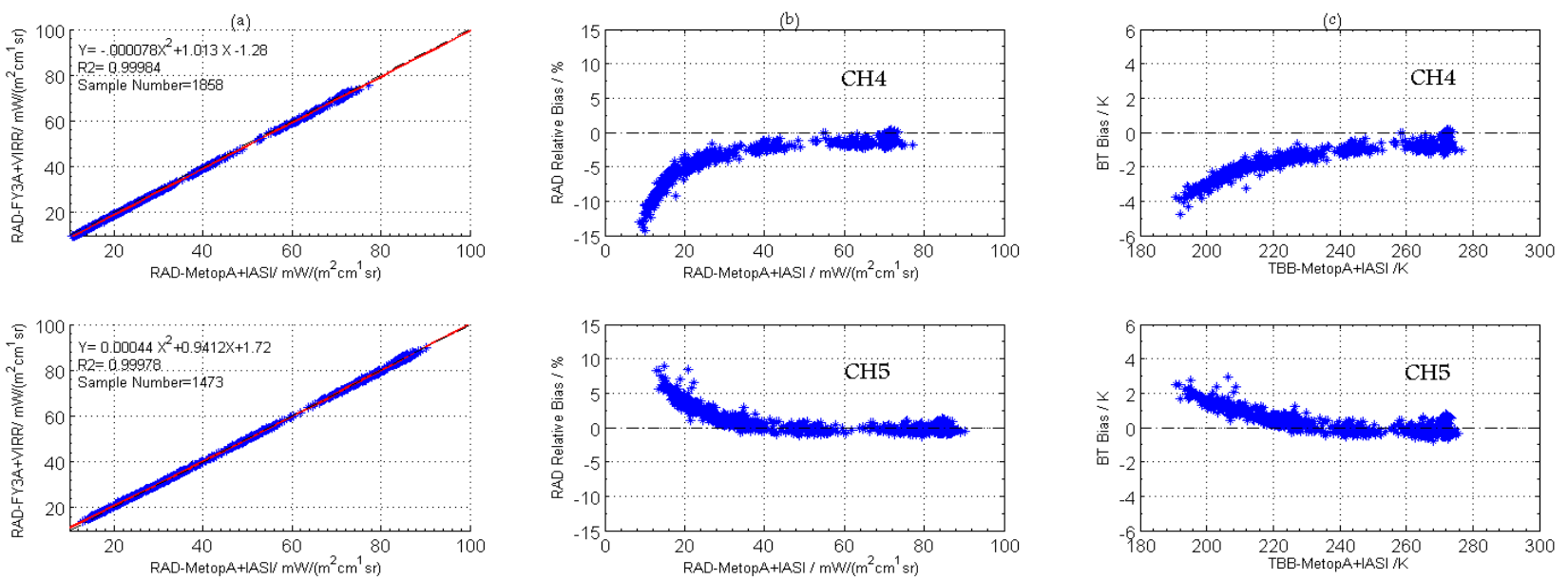

Figure 8. Radiance comparison between FY-3B/VIRR and METOP-A/IASI (a); as well as their observation differences (VIRR-IASI) in radiance (b) and BT (c) using collocation samples in August 2012. The red lines are quadratic fitting results. The top plots are for $\mathrm{CH} 4$, and those on the bottom are for $\mathrm{CH} 5$.
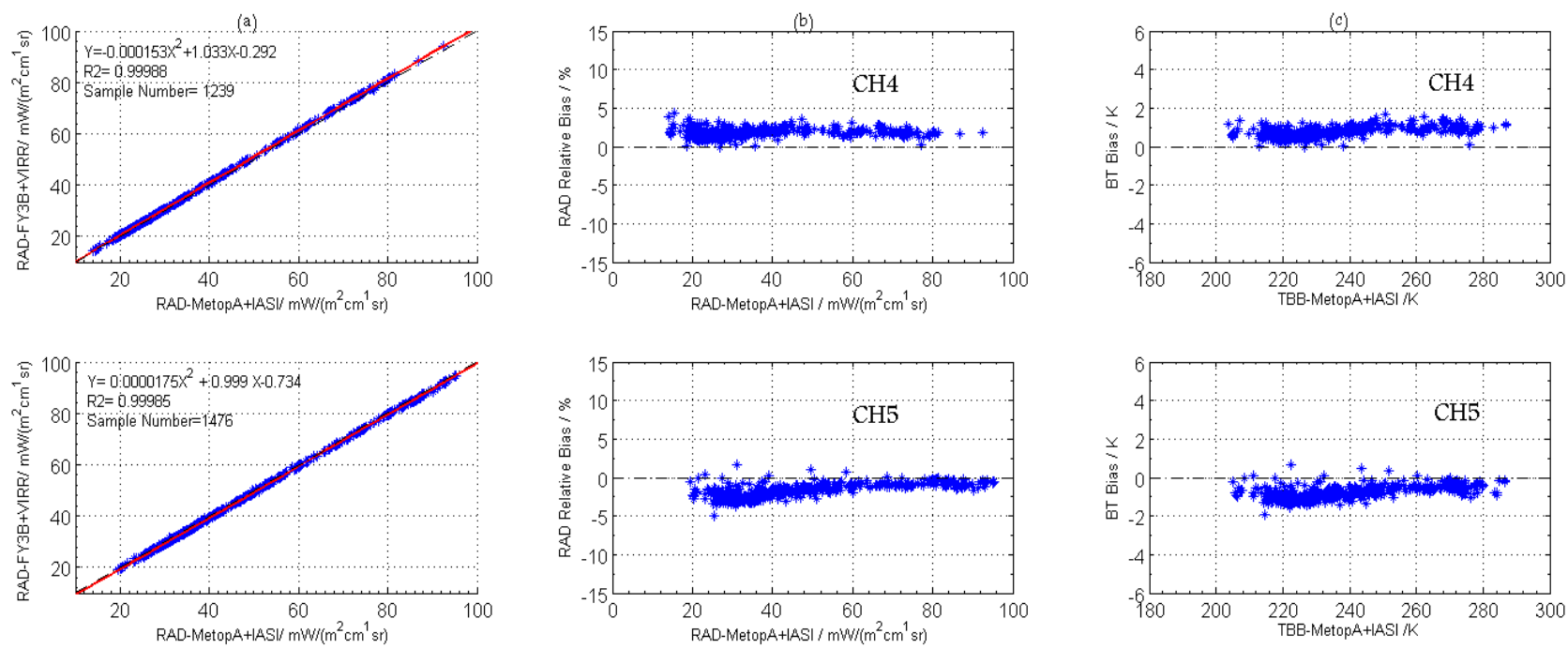
In Figure 7, both radiance and BT biases of FY-3A/VIRR in the two TIR channels are temperature-dependent. For CH4, the calibrated BTs of FY-3A/VIRR are slightly cooler than that of IASI at the range of $240 \mathrm{~K}$ to $280 \mathrm{~K}$ with an averaged bias close to $0.5 \mathrm{~K}$. BT biases increase rapidly as scene temperatures decrease. For $\mathrm{CH} 5$, the BT values are warmer than that of IASI, and the mean biases are close to zero under the warm scenes. Two reasons may cause scene temperature dependent biases, i.e., spectral response error and detector nonlinearity $[15,16]$. The spectra of VIRR CH4 and $\mathrm{CH} 5$ are located at the atmospheric window region where the radiance is less sensitive to SRF. In addition, BT biases induced by SRF error will decrease when scene temperatures decrease [16], which is inconsistent from the fact revealed in Figure 7. Therefore, the scene temperature-dependent bias appears to be primarily attributed to the nonlinearity of VIRR detectors. More evidence and correction results will be illustrated in Section 4.

Compared with FY-3A, BT measurements of FY-3B/VIRR are more consistency with IASI, and the temperature-dependent feature is less significant. Only small systematic biases are found in these two channels with a mean value of less than $1 \mathrm{~K}$. Using a similar method as that of FY-1/HRPT and AVHRR [17,18], VIRR TIR channels are calibrated as:

$$
R_{\mathrm{EV} \_ \text {LIN }}=G^{*}\left(D N_{\mathrm{EV}}-D N_{\mathrm{SV}}\right)+R_{\mathrm{SV}}
$$

where:

$$
\begin{aligned}
& G=\frac{B\left(T B B_{\mathrm{BB}}\right)-R_{\mathrm{SV}}}{D N_{\mathrm{BB}}-D N_{\mathrm{SV}}} \\
& T B B_{\mathrm{BB}}=b_{0}+b_{1} C_{\mathrm{PRT}}+b_{2} C_{\mathrm{PRT}}^{2}
\end{aligned}
$$

$R_{\mathrm{EV} \_ \text {LIN }}$ is the linear calibrated earth view (EV) radiance, and $D N_{\mathrm{EV}}$ is the earth view raw digital number. $G$ is the calibration coefficient determined on orbit based on blackbody facility (BB) and space view $(\mathrm{SV})$ measurements. $B\left(T B B_{\mathrm{BB}}\right)$ is the Plank radiance at equivalent $\mathrm{BB}$ temperature $\left(T B B_{\mathrm{BB}}\right)$ which is transformed from platinum resistance thermometer (PRT) counts using a set of static coefficients. Nonlinear correction is performed after linear radiometric calibration using Equation (2), where the correction coefficients $\left(\mathrm{a}_{0}, \mathrm{a}_{1}\right.$, and $\left.\mathrm{a}_{2}\right)$ are all determined from prelaunch laboratory measurements and remain constant over the instrument lifetime.

$$
R_{\mathrm{EV} \_\mathrm{Cor}}=a_{0}+a_{1} R_{\mathrm{EV} \_\mathrm{LIN}}+a_{2} R_{\mathrm{EV} \_ \text {LIN }}^{2}
$$

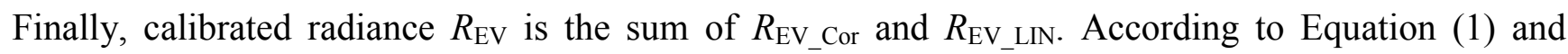
Equation (2), the systematic biases of VIRR radiometric calibration could come from the uncertainties in $T B B_{\mathrm{BB}}$ or $D N_{\mathrm{SV}}$. If $\mathrm{SV}$ measurements are contaminated, calibration coefficient will be overestimated, and positive bias will present in both channels. However, BT biases for VIRR CH5 are negative, so the space view contamination can not be the primary factor. If $T B B_{\mathrm{BB}}$ is calculated using inaccurate PRT-TBB transformation coefficients, it may cause systematic biases and behave different in different channels. However, further investigation is needed to confirm this hypothesis. 


\subsection{Seasonal Variation}

Long-term performance of VIRR TIR calibration is investigated using collocation data from January 2012 to June 2013. Figure 9 displays seasonal variations of VIRR BT biases, where the dots are monthly mean values and the short bars correspond to standard deviations (STDs). Each statistical value shown here is based on more than five hundred collocation samples.

Figure 9. Seasonal variation of BT biases in channel 4 and 5 of FY-3A\&3B VIRR versus IASI during January 2012 to June 2013, where the dot denotes mean bias, and the length of short bars indicate twice STD.
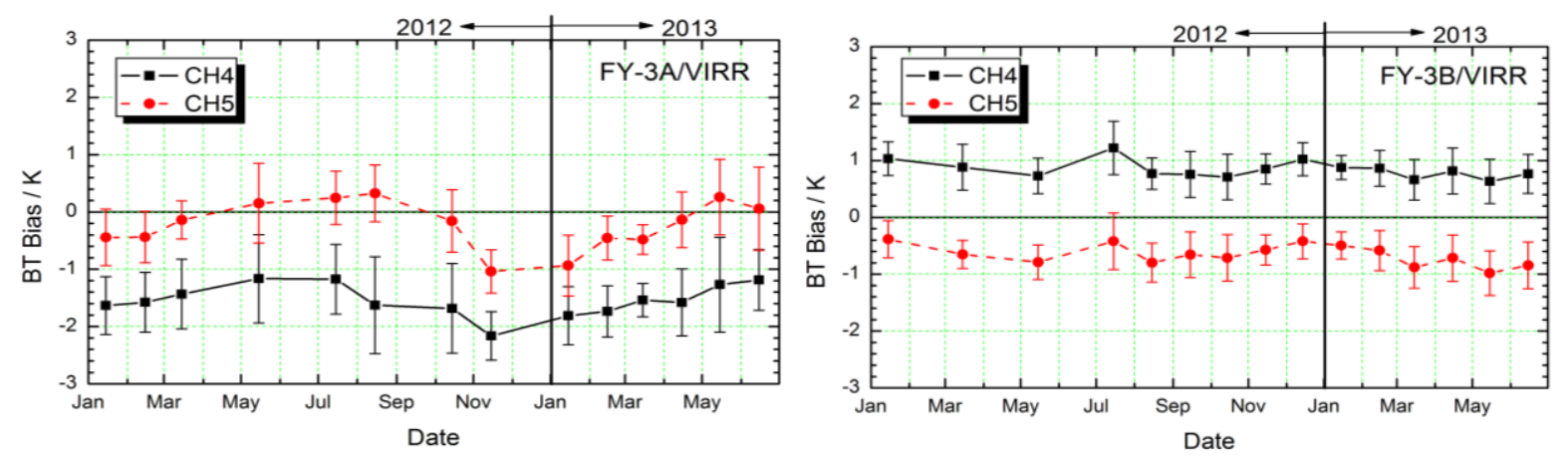

For FY-3A/VIRR, BT biases show significant seasonal variations, and the varying patterns are similar in both channels. BT biases are large in winter and decrease in summer. In CH4, VIRR BTs are much cooler than IASI with mean biases ranging from $-2 \mathrm{~K}$ to $-1 \mathrm{~K}$. Biases in $\mathrm{CH} 5$ are smaller than those in $\mathrm{CH} 4$ with monthly mean values vary from $-1 \mathrm{~K}$ to $0.2 \mathrm{~K}$. That kind of seasonal variation feature is not found from FY-3B/VIRR biases, which are much stable monthly. The standard deviations of FY-3B/VIRR biases in each month are within $0.4 \mathrm{~K}$, and differences between monthly mean biases are less than $0.5 \mathrm{~K}$. The annual mean biases are $0.84 \pm 0.16 \mathrm{~K}$ and $-0.66 \pm 0.18 \mathrm{~K}$ respectively in $\mathrm{CH} 4$ and $\mathrm{CH} 5$ of FY-3B/VIRR.

In this study, calibration assessments are based on LEO-LEO SNO samples which mostly locate at the polar area. SNO samples of FY-3A are nearby $83^{\circ} \mathrm{N}$ and $83^{\circ} \mathrm{S}$, where the onboard BB calibration is easy to suffer solar contamination [19]. When sunlight illuminates the onboard BB device, TIR calibrations will be underestimated as was illuminated in [15] and [19]. The solar illumination at TOA is largest in January and smallest in July depending on Earth-Sun distance, such that the calibration bias caused by solar contamination will also be seasonally changed. This may explain why FY-3A calibration biases are seasonal varied and become much cooler in January. However, this is just speculation, and further research is needed.

\section{Improvements to Nonlinear Correction}

To improve the nonlinear correction of the FY-3A/VIRR operational calibration program, we reevaluate the nonlinear correction coefficients in this section using the SNO intercalibration method. It can significantly decrease both temperature dependent and systematic errors. 


\subsection{Method}

Based on collocated samples, the linear calibrated VIRR radiances ( $\left.R_{\text {VIRR_LIN }}\right)$ are fitted to IASI-simulated references $\left(R S_{\text {IASI }}\right)$ using a quadratic equation, as shown in Equation (3).

$$
R S_{\mathrm{IASI}}=A_{0}+\left(A_{1}+1\right) R_{\mathrm{VIRR}_{-} L I N}+A_{2} R_{\mathrm{VIRR} \_L I N}^{2}
$$

The regression coefficients are the nonlinear correction coefficients. Table 1 lists the coefficients recalculated for the month with sufficient cold targets whose BTs are less than $220 \mathrm{~K}$. It is clear that the collocated samples used here are highly correlated and the regression coefficients in five different months show good agreement. Supposing the nonlinear feature of detectors is stable during the instrument lifetime, new correction coefficients are finally evaluated based on all samples during the five months as listed in Table 1.

Table 1. Comparison of nonlinear correction coefficients of FengYun-3 (FY-3A) Visible Infrared Radiometer (VIRR) calculated using collocation samples during different months. $A_{0}, A_{1}$ and $A_{2}$ correspond to constant, linear, and quadratic correction terms. $\mathrm{R}^{2}$ is the correlation coefficient.

\begin{tabular}{ccccccccc}
\hline & \multicolumn{9}{c}{ CH4 } & \multicolumn{4}{c}{ CH5 } \\
\cline { 2 - 9 } & $\boldsymbol{A}_{\boldsymbol{2}}$ & $\boldsymbol{A}_{\boldsymbol{I}}$ & $\boldsymbol{A}_{\boldsymbol{0}}$ & $\mathbf{R}^{\mathbf{2}}$ & $\boldsymbol{A}_{\boldsymbol{2}}$ & $\boldsymbol{A}_{\boldsymbol{1}}$ & $\boldsymbol{A}_{\boldsymbol{0}}$ & $\mathbf{R}^{\mathbf{2}}$ \\
\hline $2012-05$ & $4.0630 \mathrm{E}-04$ & $-7.0190 \mathrm{E}-02$ & 2.7916 & 0.99981 & $-1.1348 \mathrm{E}-04$ & $-3.3500 \mathrm{E}-03$ & 0.19271 & 0.99983 \\
$2012-07$ & $2.1868 \mathrm{E}-04$ & $-5.0270 \mathrm{E}-02$ & 2.3886 & 0.99689 & $-1.7219 \mathrm{E}-04$ & $1.9000 \mathrm{E}-03$ & 0.19915 & 0.99727 \\
$2012-08$ & $2.0522 \mathrm{E}-04$ & $-6.0040 \mathrm{E}-02$ & 2.7992 & 0.99991 & $-2.6655 \mathrm{E}-04$ & $5.7000 \mathrm{E}-03$ & 0.20174 & 0.99985 \\
$2013-05$ & $8.0951 \mathrm{E}-05$ & $-4.9040 \mathrm{E}-02$ & 2.5569 & 0.99906 & $-3.7013 \mathrm{E}-04$ & $1.6200 \mathrm{E}-02$ & -0.00572 & 0.99921 \\
$2013-06$ & $3.9560 \mathrm{E}-04$ & $-6.1090 \mathrm{E}-02$ & 2.5106 & 0.99943 & $-4.6927 \mathrm{E}-04$ & $4.1700 \mathrm{E}-02$ & -0.9922 & 0.99909 \\
New & $1.9639 \mathrm{E}-04$ & $-5.3780 \mathrm{E}-02$ & 2.57927 & 0.99878 & $-2.5315 \mathrm{E}-04$ & $8.0900 \mathrm{E}-03$ & 0.09126 & 0.99887 \\
Operational & $3.8094 \mathrm{E}-04$ & $-6.2200 \mathrm{E}-02$ & 1.59565 & & $3.4763 \mathrm{E}-04$ & $-6.4250 \mathrm{E}-02$ & 1.95424 & \\
\hline
\end{tabular}

\subsection{Improvement of New Nonlinear Correction}

Using a similar method as in Section 3, improvements of the new nonlinear correction for FY-3A/VIRR are assessed. Figure 10 shows BT biases of FY-3A/VIRR TIR channels in August 2012, using new nonlinear coefficients. Compared with Figure 7, the scene temperature-dependent biases are greatly improved, and the systematic biases are also reduced with a mean bias close to zero. BT biases in other months are also investigated, and their normalized probability distribution functions (PDFs) are displayed in Figure 11. In CH4, PDFs of BT biases without nonlinear correction or corrected using operational coefficients show multimodal patterns, and the peak biases are all negative. Under the new nonlinear correction, PDFs are nearly normal distributions, and the peak biases approach zero. The new nonlinear correction in $\mathrm{CH} 5$ reduces the probability of side lobes which are mostly attributed to nonlinear biases. The positive effect of the new nonlinear coefficients to BT measurements verifies that the scene temperature-dependent bias revealed in Figure 7 is primarily caused by inaccurate nonlinear correction. 
Figure 10. BT biases of FY-3A/VIRR, in August 2012, vs. IASI (VIRR-IASI) using new nonlinear correction coefficients. The solid lines are linear regression results as a function of temperature.
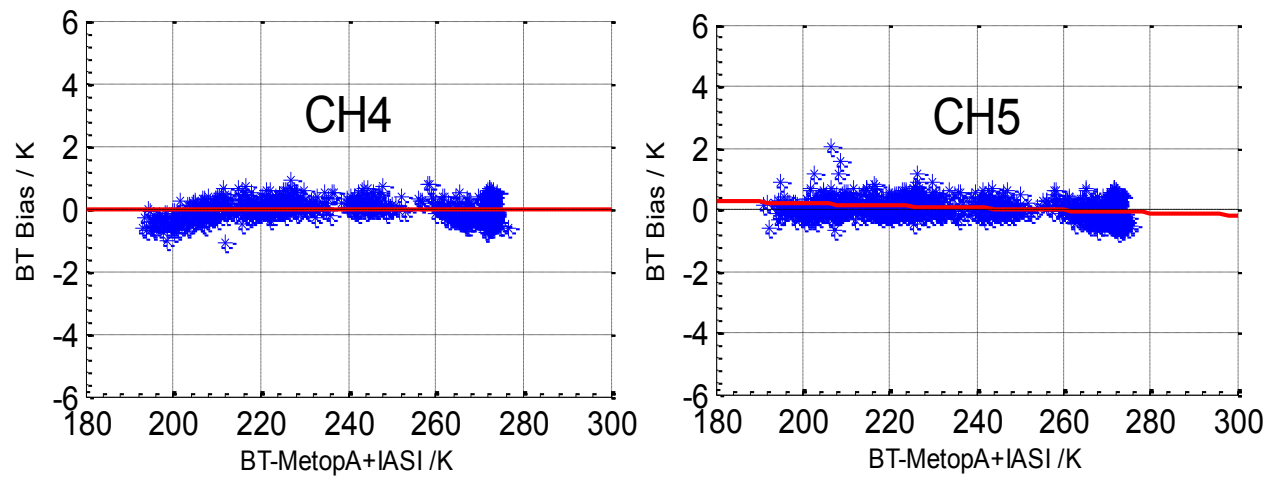

Figure 11. Normalized PDFs of FY-3A/VIRR BT biases in different months. The legends Uncorrected, Operational, and NewCorrected, respectively, indicate the calibrated BTs without nonlinear correction, with operational correction, and with new correction. The top figures are for channel 4, and the bottom figures are for channel 5.
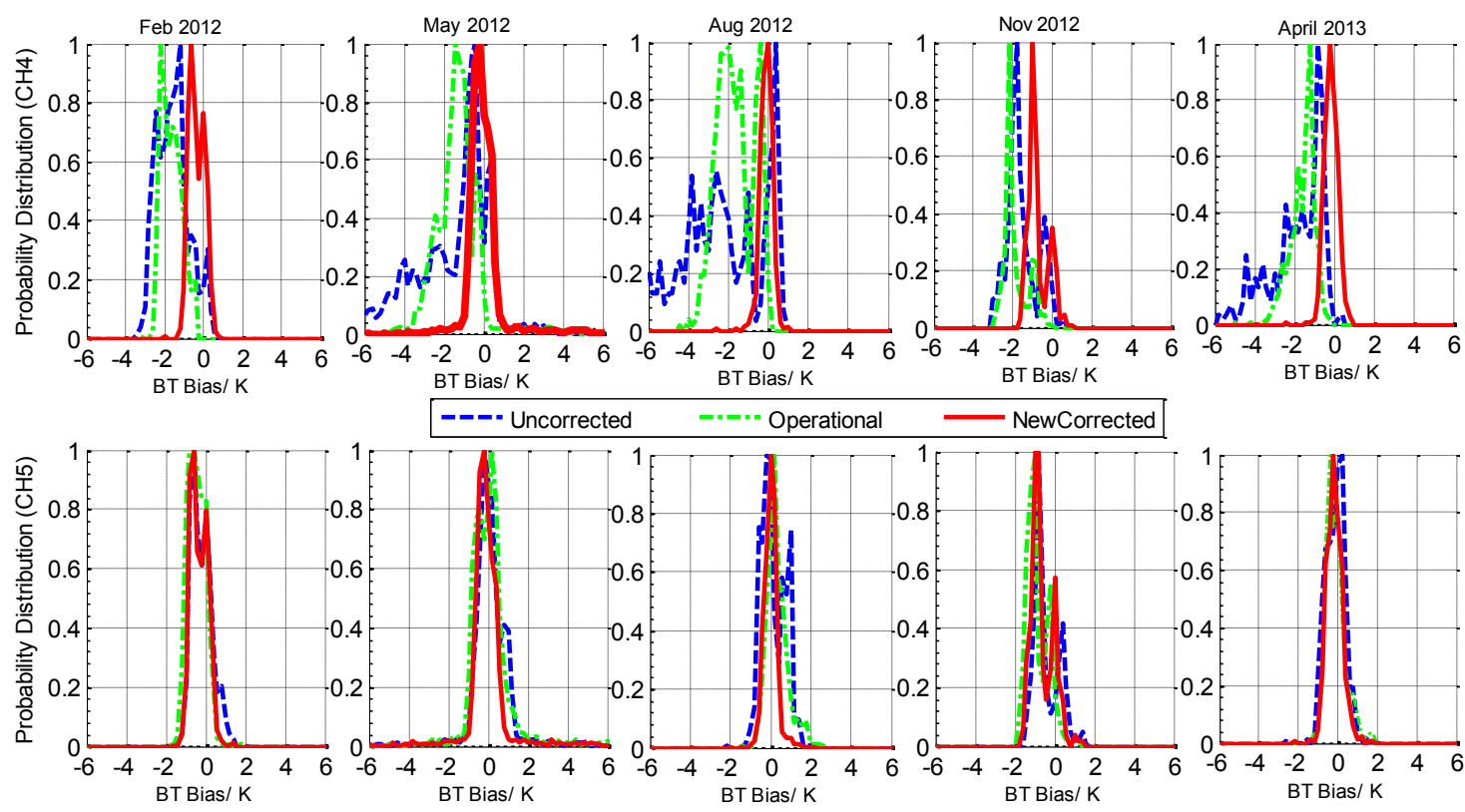

\section{Conclusions}

Based on the Low Earth Orbit (LEO)-LEO simultaneous nadir overpass (SNO) intercalibration method, the radiometric calibration accuracy and its long-term variation of FengYun-3 (FY-3)A\&B Visible Infrared Radiometer (VIRR) in thermal infrared bands are assessed. Infrared Atmosphere Sounding Instrument (IASI) onboard METOP-A is chosen as the transfer reference, which is a well-calibrated hyperspectral radiometer and recommended by the Global Space-based Intercalibration System (GSICS). Observations from VIRR thermal infrared bands are compared with simulated radiances derived from convolved hyperspectral measurements with VIRR spectral response functions (SRFs). Assessments are based on various near-simultaneous nadir observations under a set of 
collocation criteria in space, temporal, and viewing geometries. Uncertainties of the intercalibration method used here are analyzed by investigating the correlation between BT differences and all collocation factors. Our results indicate that there is no dependent relationship and the method uncertainty is negligible.

BT biases of VIRR vs. IASI are analyzed using SNO collocated samples from January 2012 to June 2013. According to our study, the following conclusions can be drawn:

(1) Statistically, BTs observed from FY-3A/VIRR are cooler than those from IASI. Monthly mean biases range from $-2 \mathrm{~K}$ to $-1 \mathrm{~K}$ for channel $(\mathrm{CH}) 4$ and from $-1 \mathrm{~K}$ to $0.2 \mathrm{~K}$ for $\mathrm{CH} 5$ in 2012 . Observations from FY-3B/VIRR are much consistent with those from IASI, and the annual mean biases are $0.84 \pm 0.16 \mathrm{~K}$ and $-0.66 \pm 0.18 \mathrm{~K}$ for $\mathrm{CH} 4$ and $\mathrm{CH} 5$, respectively.

(2) BT biases of FY-3A/VIRR are temperature dependent in both thermal infrared channels, which are shown to be attributed to the inaccurate nonlinear correction of VIRR detectors. This characteristic is not found from FY-3B/VIRR biases.

(3) Monthly mean biases of FY-3A/VIRR show significant seasonal variations in both thermal infrared channels with similar varying pattern. The BT biases reach the minimum from May to July, and increase to the maximum from November to December. This characteristic is also not found from FY-3B/VIRR biases.

The nonlinear character of FY-3A/VIRR is also studied in this paper. Correction coefficients are reevaluated using SNO collocation samples. Verification results indicate that under the new nonlinear correction, the scene temperature dependent and systematic biases are greatly reduced. Intercalibration, based on hyperspectral measurements, provides a flexible technique to evaluate and correct calibration bias on-orbit. However, LEO-LEO SNO samples mostly locate at the polar area, where the surface type is limited. In the future, the collocation should be extended to the equator region by relaxing the temporal and spatial restriction, and the spatial distribution character of calibration biases should be studied.

\section{Acknowledgments}

This work has been co-supported by National Key Basic Research and Development Program (973 Program) (2011CB403401), National High Technology Research and Development Program (863 Program) (2012AA120903-01), China's Research and Development Special Fund for Public Welfare Industry (Meteorology GYHY201206002), and National Natural Science Foundation of China (41105010).

\section{Author Contributions}

$\mathrm{Na} \mathrm{Xu}$ and Lin Chen carried out the data analyses. Liyang Zhang made onboard calibration calculations and analyses. Xiuqing Hu and Peng Zhang gave important suggestions on bias analyses. $\mathrm{Na} \mathrm{Xu}$, Xiuqing $\mathrm{Hu}$ and Lin Chen wrote the manuscript.

\section{Conflicts of Interest}

The authors declare no conflict of interest. 


\section{References}

1. Dong, C.H.; Yang, J.; Zhang, W.J.; Lu, N., Shi, J.; Zhang, P.; Liu, Y.; Cai, B. An overview of a new Chinese weather satellite FY-3A. Bull. Am. Meteorol. Soc. 2009, 90, 1531-1544.

2. Chander, G.; Hewison, T.J.; Nigel, F. Overview of intercalibration of satellite instruments. IEEE Trans. Geosci. Remote Sens. 2013, 51, 1056-1080.

3. Wu, X.; Goldberg, M.D. Global space-based intercalibration system (GSICS). Proc. SPIE 2007, 6684, doi:10.1117/12.735246.

4. Wu, X.; Hewison, T.; Tahara, Y. GSICS GEO-LEO intercalibration: Baseline algorithm and early results. Proc. SPIE 2009, 7456, 745604-1-745604-12.

5. Hewison, T.; Wu, X.; Yu, F.; Tahara, Y.; Hu, X.; Kim, D.; Koenig, M. GSICS intercalibration of infrared channels of geostationary imagers using Metop/IASI. IEEE Trans. Geosci. Remote Sens. 2013, 51, 1056-1080.

6. Aumann, H.H; Broberg, S.; Elliott, D.; Gaiser, S.; Gregorich, D. Three years of Atmospheric Infrared Sounder radiometric calibration validation using sea surface temperatures. J. Geophys. Res. 2006, 111, doi:10.1029/2005JD006822.

7. Blumstein, D.; Tournier, B.; Cayla, F.R.; Phulpin, T.; Fjortoft, R.; Buil, C.; Ponce, G. In-flight performance of the Infrared Atmospheric Sounding Interferometer (IASI) on METOP-A. Proc. SPIE 2007, 6684, doi:10.1117/12.734162.

8. Wang, L.; Goldberg, M.; Wu, X.; Cao, C.; Iacovazzi, R.; Yu, F.; Li, Y. Consistency assessment of Atmospheric Infrared Sounder and Infrared Atmospheric Sounding Interferometer radiance: Double differences versus simultaneous NADIR overpass. J. Geophys. Res. 2011, 116, doi:10.1029/2010JD014988.

9. Xu, N.; Hu, X.Q.; Chen, L.; Min, M. Intercalibration of infrared channels of FY-2/VISSR using highspectral resolution sensors IASI and AIRS. J. Remote Sens. 2012, 16, 939-952. (In Chinese with English Abstract)

10. Hu, X.Q.; Xu, N.; Weng, F.Z.; Zhang, Y.; Chen, L.; Zhang, P. Long-term monitoring and correction of FY-2 infrared channel calibration using AIRS and IASI. IEEE Trans. Geosci. Remote Sens. 2013, 51, 5008-5018.

11. Wang, L. Intercalibration of GOES-11 and GOES-12 water vapor channels with MetOp IASI hyperspectral measurements. J. Atmos. Ocean. Technol. 2009, 26, 1843-1855.

12. Yu, F.F.; Wu, X.Q. Radiometric calibration accuracy of GOES sounder infrared channels. IEEE Trans. Geosci. Remote Sens. 2013, 51, 1187-1199.

13. Hewison, T.J. An evaluation of the uncertainty of the GSICS SEVIRI-IASI intercalibration products. IEEE Trans. Geosci. Remote Sens. 2013, 51, 1171-1181.

14. Tahara, Y. New approach to intercalibration using high spectral resolution sounder. Meteorol. Satell. Center Techn. Note 2008, 50, 1-14.

15. Walton, C.C.; Sullivan, J.T.; Rao, C.R.N.; Weinreb, M.P. Corrections for detector nonlinearities and calibration inconsistencies of the infrared channels of the advanced very high resolution radiometer. J. Geophys. Res. 1998, 103, 3323-3337.

16. Wu, X.Q.; Yu, F.F. Correction for GOES imager spectral response function using GSICS. Part I: Theory. IEEE Trans. Geosci. Remote Sens. 2013, 51, 1215-1223. 
17. Cracknell, A.P. The Advanced Very High Resolution Radiometer; Taylor \& Francis: London, UK, 1997.

18. NOAA KLM User's Guide (September 2000 Revision). Available online: http://www.ncdc.noaa.gov/oa/pod-guide/ncdc/docs/klm/index.htm (accessed on 5 May 2006).

19. Cao, C.; Weinreb, M.; Sullivan, J. Solar contamination effects on the infrared channels of the Advanced Very High Resolution Radiometer (AVHRR). J. Geophys. Res. 2001, 106, $33463-33469$.

(C) 2014 by the authors; licensee MDPI, Basel, Switzerland. This article is an open access article distributed under the terms and conditions of the Creative Commons Attribution license (http://creativecommons.org/licenses/by/3.0/). 